\title{
ANGULAR VELOCITY OF ROTATION OF EXTENDED BODIES IN GENERAL RELATIVITY
}

\author{
S.A. KLIONER \\ Institute of Applied Astronomy \\ 197042 St.Petersburg, Russia
}

\begin{abstract}
We consider rotational motion of an arbitrarily composed and shaped, deformable weakly self-gravitating body being a member of a system of $N$ arbitrarily composed and shaped, deformable weakly selfgravitating bodies in the post-Newtonian approximation of general relativity. Considering importance of the notion of angular velocity of the body (Earth, pulsar) for adequate modelling of modern astronomical observations, we are aimed at introducing a post-Newtonian-accurate definition of angular velocity. Not attempting to introduce a relativistic notion of rigid body (which is well known to be ill-defined even at the first post-Newtonian approximation) we consider bodies to be deformable and introduce the postNewtonian generalizations of the Tisserand axes and the principal axes of inertia.
\end{abstract}

\section{Introduction}

In the framework of general relativity the spin (angular momentum) of an arbitrarily composed and shaped, weakly self-gravitating deformable body being a member of a system of $\mathrm{N}$ arbitrarily composed and shaped, weakly self-gravitating deformable bodies is a well-defined notion (see, e.g., Damour, Soffel, Xu, 1993). However, from the astronomical (observational) point of view not only the spin, but also the angular velocity of rotation of celestial bodies plays an important role. Indeed, it is the angular velocity of the Earth's rotation which is directly related to the observable quantities in the modern geodynamical observational techniques (VLBI, SLR, LLR, GPS/GLONASS). On the other hand, the angular velocity of rotation of pulsars plays the primary role in modelling pulsar timing data. Permanently 
increasing accuracy of geodynamical and pulsar timing observations makes it very important to have a relativistic definition of angular velocity of real bodies as well as relativistic equations describing time-dependence of the angular velocity.

For the first time, rotational equations of motion of extended bodies in general relativity have been discussed by Fock (1959). Fock succeeded to derive at the first post-Newtonian level the rotational equations of motion of an extended body being a member of a system of $\mathrm{N}$ gravitationally interacting extended bodies. Later the equations derived by Fock have been thoroughly investigated in Brumberg (1972) assuming the rigid-body distribution of the velocity of the matter of each body relative to a global reference system. Recently the result has been significantly improved by Damour, Soffel and Xu (1993). In that paper the post-Newtonian rotational equations of motion of an arbitrarily shaped, weakly self-gravitating bodies being members of a system of $\mathrm{N}$ bodies have been derived. Considering each body in its own local reference system the authors showed how to define a post-Newtonian spin vector of a body whose local-time evolution can be entirely expressed through bilinear couplings between the BlanchetDamour (BD) multipole moments of that body and the tidal moments of the external gravitational field it experiences.

In order to introduce the post-Newtonian angular velocity we have to understand how the post-Newtonian spin vector can be split into a product of a tensor of inertia and an angular velocity. This problem, being quite trivial in Newtonian physics, is nontrivial and, so far, unsolved problem in the framework of general relativity (see, Thorne, Gürsel, 1983; Soffel, 1994). Until now virtually all papers dealing with the problem in question were devoted to relativistic generalizations of the rigid-body rotation. It is quite understandable since the coupling between the relativistic effects in the rotational motion of the Earth or a pulsar and the effects of their non-rigidity seem to be much smaller than the relativistic effects themselves which were the primary goal of the investigations. However, in the relativistic framework it is impossible to define rigorously a precessing, rigidly rotating body even at the first post-Newtonian approximation (see, e.g., Thorne, Gürsel, 1983; Soffel, 1994 and references cited therein). That is why, the authors were forced to drop all the terms nonlinear with respect to angular velocity which might be a critical restriction. Moreover, it seems to be unsatisfactory to introduce, into relativistic considerations, a notion which cannot be rigorously defined even in the first post-Newtonian approximation.

In Newtonian case the notion of rigid body plays a fundamental role in discussing the spatial rotation of extended bodies. Rigid-body rotation is not only a first-order (though usually quite accurate) approximation for real celestial bodies, but it also intimately relates to the properties of the 
absolute Newtonian space which is an Euclidean 3-dimensional manifold from the mathematical point of view. However, it is well known what to do if the body under consideration cannot be considered as rigid: we have to consider velocity distribution of the matter inside the body and split the velocity field into a rigid rotation and a deformation (see, e.g., Moritz, Mueller, 1987). In this way we can introduce the so-called Tisserand axes (a rigidly rotating reference system with respect to which the Newtonian angular momentum of the body vanishes identically) or the principal axes of inertia (a rigidly rotating reference system in which the Newtonian tensor of inertia is diagonal at any moment of time). Both these rigidly rotating reference systems allow one to define the angular velocity of a non-rigid body which has some definite physical meaning. This angular velocity is not in fact directly related to the body. It is the angular velocity of rotation of some rigidly rotating reference system (in which the body appears to be nonrotating in one sense or another) with respect to the corresponding Newtonian inertial reference system.

In analogy to Newtonian physics it is quite natural idea to consider deformable bodies in general relativity. However, the first and probably the only paper dealing with rotation of non-rigid bodies in general relativity is Voinov (1988). The principal idea is to consider relativistic effects in internal motions within the body as additional deformations which can be treated in analogy to Newtonian deformations. Unfortunately, because of several unjustified (or, sometimes, unnecessary) assumptions the paper can be considered only as a preliminary one. Our aim is to follow the principal idea formulated above as rigorously as possible, and generalize the Newtonian concepts of the Tisserand axes and the principal axes of inertia onto the post-Newtonian approximation of general relativity without any attempt to introduce the notion of a post-Newtonian rigid body.

\section{Relativistic reference systems}

Although it is well known that in the framework of general relativity any reference systems covering the space-time region under consideration are mathematically equivalent, one can prefer one reference system or another to perform actual calculations. The basic reason for the preference is physical adequacy (or convenience) of a reference system for the problem under consideration. In fact, the choice of an adequate reference system is an important part of solving any problem. Recently it has been shown that to describe adequately physics within an isolated system of $\mathrm{N}$ gravitating bodies one should use several relativistic reference systems: one global reference system covering all the bodies simultaneously and one local reference system for each of the bodies (see, e.g., Brumberg, Kopejkin (1989), Kopejkin 
(1988) and Damour, Soffel, Xu (1991), (1992), (1993)).

The global reference system is suitable for describing relative motion of the bodies. We will designate it as BRS (Barycentric Reference System). The local reference systems are physically adequate for describing local physical processes related to the body under consideration. The local reference systems are proved to be suitable to describe rotational motion of the individual bodies (Damour, Soffel, Xu, 1993). Bearing in mind that the Earth is the primary body whose motion and rotation is to be investigated we will designate the local reference system of the body under consideration as GRS (Geocentric Reference System), although "GRS" can be related not only to the Earth, but to any other body.

We assume general continuous distribution of the matter having the energy-momentum tensor $T^{\alpha \beta}$. According to the usual post-Newtonian assumptions for the matter

$$
T^{00}=\mathcal{O}\left(c^{2}\right), \quad T^{0 i}=\mathcal{O}(c), \quad T^{i j}=\mathcal{O}(1) .
$$

We also suppose that the matter is localized in $\mathrm{N}$ separate blobs (bodies) and we discuss rotation of one of them.

Not going into complicated details of constructing the global and local reference systems (see, e.g., Brumberg, Kopejkin (1989); Kopejkin (1988); Damour, Soffel, Xu (1991); Klioner, Voinov (1993) for details), let us write down the generic form of the metric tensor which is valid for both the BRS and the GRS

$$
\begin{aligned}
g_{00} & =1-\frac{2}{c^{2}} W+\frac{2}{c^{4}}\left(W^{2}-\chi_{, 00}-a_{, 0}\right)+\mathcal{O}\left(c^{-6}\right), \\
g_{0 i} & =\frac{1}{c^{3}}\left(4 U^{i}-a_{, i}\right)+\mathcal{O}\left(c^{-5}\right), \\
g_{i j} & =-\delta_{i j}\left(1+\frac{2}{c^{2}} W\right)+\mathcal{O}\left(c^{-4}\right) .
\end{aligned}
$$

Here, as usual, comma denotes a partial derivative (so that, $a_{, 0}=\frac{\partial}{\partial t} a$ and $\left.a_{, i}=\frac{\partial}{\partial x^{i}} a\right), \delta^{i j}$ is the Kronecker symbol and $a=a(t, \mathbf{x})$ is an arbitrary function which parameterizes a class of coordinate gauges allowed by our formalism and can be interpreted as a transformation of time $\tilde{t}=t+c^{-4} a$. This function can be left unspecified since the post-Newtonian equations of motion (both translational and rotational) are well known to be independent of $a$. Note that $a=0$ corresponds to the harmonic gauge, while $a=-\chi, 0$ leads to the standard PPN (isotropic) gauge. The post-Newtonian potential $W$, the vector potential $U^{i}$, and the superpotential $\chi$ can be derived from the Einstein equations reduced with the corresponding gauge conditions

$$
W_{, i i}=-4 \pi G \sigma, \quad U_{, j j}^{i}=-4 \pi G \sigma^{i}, \quad \chi_{, i i}=W,
$$


where following Damour, Soffel and Xu (1991) we designate

$$
\sigma=\frac{1}{c^{2}} T^{\alpha \alpha}, \quad \sigma^{i}=\frac{1}{c} T^{0 i} .
$$

Equations (3) should be solved with the account for specific boundary conditions which are to be chosen differently in the BRS and the GRS (see, e.g., Kopejkin, 1988; Damour, Soffel, Xu, 1991). In both the BRS and the GRS the potentials $W, U^{i}$ and $\chi$ can be split into parts generated by the body under consideration and the external potentials induced by the rest of the matter (and, in case of the GRS, by inertial forces)

$$
W=W_{\mathrm{int}}+W_{\mathrm{ext}}, \quad U^{i}=U_{\mathrm{int}}^{i}+U_{\mathrm{ext}}^{i}, \quad \chi=\chi^{\mathrm{int}}+\chi^{\mathrm{ext}} .
$$

\section{Post-Newtonian rotational equations of motion}

Let us consider a reference system $(t, \mathbf{x})$ which can be either the BRS or the GRS. We will call this reference system RS. There are several ways to derive the post-Newtonian rotational equations of motion of an extended body relative to the RS. One can mention the Fock approach and the LandauLifshitz one. The former consists in evaluating the following integral over the support $V$ of the body under consideration

$$
\varepsilon_{i j k} \int_{V}(-g) x_{; \beta}^{j} T_{; \beta}^{k \beta} d x^{3}=0
$$

which vanishes due to the local equations of motion of the matter

$$
T_{; \beta}^{\alpha \beta}=0 \text {. }
$$

Here semicolon denotes the covariant derivative,

$$
-g=1+\frac{1}{c^{2}} 4 W+\mathcal{O}\left(c^{-4}\right)
$$

is the determinant of the metric tensor $g_{\alpha \beta}$ defined by (2), and $\varepsilon_{i j k}$ is the fully antisymmetric Levi-Civita symbol $\left(\varepsilon_{123}=+1\right)$.

The Landau-Lifshitz approach is based on the use of the so-called Landau-Lifshitz pseudotensor $t^{\alpha \beta}$ of energy-momentum of gravitational field defined in such a way that (see, e.g., Landau, Lifshitz (1971))

$$
\left((-g)\left(T^{\alpha \beta}+t^{\alpha \beta}\right)\right)_{, \beta}=0 \text {. }
$$

Then one can derive the rotational equations of motion from the integral

$$
\varepsilon_{i j k} \int_{V} x^{j}\left((-g)\left(T^{k \beta}+t^{k \beta}\right)\right)_{, \beta} d x^{3}=0
$$


Both approaches mentioned above result in the same post-Newtonian rotational equations of motion which can be written as (details will be published elsewhere)

$$
\frac{d}{d t} S^{i}=F^{i}+\mathcal{O}\left(c^{-4}\right)
$$

Here $S^{i}$ is the post-Newtonian spin which can be written as a well-defined compact support integral

$$
\begin{gathered}
S^{i}=\varepsilon_{i j k} \int_{V} x^{j} Q^{k} d x^{3}+\mathcal{O}\left(c^{-4}\right) \\
Q^{k}=\sigma^{k}\left(1+\frac{4}{c^{2}} W\right)-\frac{1}{2 c^{2}} G \sigma \int_{V} \sigma^{s}\left(t, \mathbf{x}^{\prime}\right) \frac{7 \delta^{k s}+n^{k} n^{s}}{\left|\mathbf{x}-\mathbf{x}^{\prime}\right|} d x^{3}+\mathcal{O}\left(c^{-4}\right) \\
n^{i}=\frac{x^{i}-x^{i}}{\left|\mathbf{x}-\mathbf{x}^{\prime}\right|}
\end{gathered}
$$

Now let us assume the following relation between the components of the energy-momentum tensor, which, in fact, is valid under very general assumptions on the matter (see, e.g., Fock, 1959)

$$
(-g) \sigma^{i}=\left(\rho^{*} \delta^{i j}+\frac{1}{c^{2}} p^{i j}\right) \dot{x}^{j}+\mathcal{O}\left(c^{-4}\right), \quad \rho^{*}=(-g) \frac{1}{c^{2}} T^{00},
$$

where $p^{i j}$ is the stress tensor of the matter. Then we get

$$
Q^{k}=\rho^{*} \dot{x}^{k}+\frac{1}{c^{2}}\left(p^{k s} \dot{x}^{s}-\frac{1}{2} G \rho^{*} \int_{V} \rho^{*}\left(t, \mathbf{x}^{\prime}\right) \dot{x}^{\prime s} \frac{7 \delta^{k s}+n^{k} n^{s}}{\left|\mathbf{x}-\mathbf{x}^{\prime}\right|} d x^{\prime 3}\right)+\mathcal{O}\left(c^{-4}\right) .
$$

On the right-hand side of (11) we have a post-Newtonian torque $F^{i}$ defined as

$$
\begin{aligned}
& F^{i}=\varepsilon_{i j k} \int_{V} \sigma(t, \mathbf{x}) x^{j} f^{k} d x^{3}+\mathcal{O}\left(c^{-4}\right), \\
& f^{k}=W_{, k}^{\text {ext }}+\frac{1}{c^{2}}\left(4 \dot{U}_{\text {ext }}^{k}+\dot{\chi}_{, 0 k}^{\text {ext }}-\dot{x}^{s}\left(4 U_{\text {ext }, k}^{s}+\chi_{, 0 k s}^{\text {ext }}\right)\right)
\end{aligned}
$$

Note that $f^{k}$ is proportional to the external potentials, and therefore, in analogy to Newtonian case the post-Newtonian torque $F^{i}$ vanishes for isolated bodies $\left(W_{\text {ext }}=0, U_{\text {ext }}^{i}=0, \chi^{\text {ext }}=0\right)$.

It is important to note also that casting the integrals (6) or (10) into the form (11) is not unique. For example, the equations of rotational motion which we quoted above do not coincide with those derived in Brumberg (1972) where a part of our post-Newtonian torque $F^{i}$ have been moved to the left-hand side of (11) and interpreted as a part of the post-Newtonian 
spin $S^{i}$. However, Eqs. (11)-(17) allow us to re-write the post-Newtonian spin density $Q^{k}$ in the form (13)-(16) which makes it (and, therefore, $S^{i}$ itself) explicitly proportional to $\sigma^{i}$ and therefore to the velocity $\dot{x}^{i}$ of the matter). The latter circumstance will be important for further considerations. Note also that our form of the rotational equations of motion (11)(17) being written for a Damour-Soffel-Xu local reference system coincides with Eq. (2.4)-(2.8) of Damour, Soffel, Xu (1993).

\section{Rotating reference system}

According to the basic idea exposed in Introduction we have to define a relativistic rotating reference system (let us designate it as $\mathrm{RS}^{+}$and its time and space coordinates as $\left.\left(\bar{t}, \bar{x}^{i}\right)\right)$. The $\mathrm{RS}^{+}$has to be a post-Newtonian generalization of the Newtonian Tisserand axes (or the Newtonian principal axes of inertia). This implies that $\bar{t}=t+\mathcal{O}\left(c^{-2}\right)$, and $\bar{x}^{i}=P^{i j}(t) x^{j}+\mathcal{O}\left(c^{-2}\right)$, where $P^{i j}(t)$ is a time-dependent orthogonal matrix. Generally speaking, we could add some post-Newtonian pieces $\mathcal{O}\left(c^{-2}\right)$ into the transformations. However, considering that it is unclear how to introduce relativistically meaningful macroscopic spatial rotation in curved space-time of general relativity, it is quite reasonable to define the coordinate transformations between the $\mathrm{RS}^{+}$and the $\mathrm{RS}$ as a rigid Newtonian spatial rotation

$$
\begin{gathered}
\bar{t}=t, \\
\bar{x}^{i}=P^{i j}(t) x^{j} .
\end{gathered}
$$

Here the rotation has the meaning of an Euclidean 3-dimensional timedependent rotation in the 3 -space formed by the spatial coordinates of the RS. Using (18) one can easily derive the metric tensor $\bar{g}^{\alpha \beta}$ of the $\mathrm{RS}^{+}$, the relations between the components of the stress-energy tensor $\bar{T}^{\alpha \beta}$ in the $\mathrm{RS}^{+}$and those in the RS, etc.

\section{Rotational equations of motion relative to the $\mathrm{RS}^{+}$}

There are several ways to derive the rotational equations of motion of an extended body relative to the $\mathrm{RS}^{+}$. Let us mention

- the Fock approach (Eq. (6) written in the $\mathrm{RS}^{+}$);

- the Landau-Lifshitz approach (Eq. (10) written in the $\mathrm{RS}^{+}$);

- transforming the rotational equations of motion relative to the RS (11)-(17) into those relative to the $\mathrm{RS}^{+}$with the aid of the coordinate transformations (18). 
All the approaches result in the same rotational equations of motion relative to the rigidly rotating reference system $\mathrm{RS}^{+}$

$$
\frac{d}{d t}\left(\bar{S}^{i}+\bar{C}^{i j} \bar{\omega}^{j}\right)+\varepsilon_{i j k} \bar{\omega}^{j}\left(\bar{S}^{k}+\bar{C}^{k s} \bar{\omega}^{s}\right)=P^{i j} F^{j}+\mathcal{O}\left(c^{-4}\right)
$$

where

$$
\bar{\omega}^{i}=\frac{1}{2} \varepsilon_{i j k} \dot{P}^{j m} P^{k m}
$$

is the angular velocity of rotation of the $\mathrm{RS}^{+}$relative to the RS projected onto the axes of the $\mathrm{RS}^{+}$,

$$
\begin{gathered}
\bar{S}^{i}=\varepsilon_{i j k} \int_{\bar{V}} \bar{x}^{j} \bar{Q}^{k} d \bar{x}^{3}+\mathcal{O}\left(c^{-4}\right) \\
\bar{Q}^{k}=\rho^{*} \dot{\bar{x}}^{k}+\frac{1}{c^{2}}\left(\bar{p}^{k s} \dot{\bar{x}}^{s}-\frac{1}{2} G \rho^{*} \int_{\bar{V}} \rho^{*}\left(t, \overline{\mathbf{x}}^{\prime}\right) \dot{\bar{x}}^{\prime s} \frac{7 \delta^{k s}+\bar{n}^{k} \bar{n}^{s}}{\left|\overline{\mathbf{x}}-\overline{\mathbf{x}}^{\prime}\right|} d \bar{x}^{\prime 3}\right)+\mathcal{O}\left(c^{-4}\right) \\
\rho^{*}=\frac{1}{c^{2}}(-\bar{g}) \bar{T}^{00}=\frac{1}{c^{2}}(-g) T^{00} \\
\bar{p}^{i j}=P^{i k} P^{j s} p^{k s} \\
\bar{n}^{i}=P^{i j} n^{j}=\frac{\bar{x}^{i}-\bar{x}^{i}}{\left|\overline{\mathbf{x}}-\overline{\mathbf{x}}^{\prime}\right|} \\
\bar{C}^{i j}(t)=\int_{\bar{V}} \rho^{*}\left(\delta^{i j} \bar{x}^{s} \bar{x}^{s}-\bar{x}^{i} \bar{x}^{j}\right) d \bar{x}^{3}+\frac{1}{c^{2}} \varepsilon_{i a k} \varepsilon_{j b s} \int_{\bar{V}} \bar{x}^{a} \bar{x}^{b} \bar{p}^{k s} d \bar{x}^{3} \\
-\frac{G}{2 c^{2}}\left(7 \delta^{i j} \bar{\alpha}^{s s}-7 \bar{\alpha}^{i j}+\bar{\beta}^{i j}\right)+\mathcal{O}\left(c^{-4}\right) \\
\bar{\alpha}^{i j}(t)=\int_{\bar{V}} \int_{\bar{V}} \rho^{*}(t, \overline{\mathbf{x}}) \rho^{*}\left(t, \overline{\mathbf{x}}^{\prime}\right) \frac{\bar{x}^{i} \bar{x}^{\prime j}}{\left|\overline{\mathbf{x}}-\overline{\mathbf{x}}^{\prime}\right|} d \bar{x}^{\prime 3} d \bar{x}^{3} \\
\bar{\beta}^{i j}(t)=\int_{\bar{V}} \int_{\bar{V}} \rho^{*}(t, \overline{\mathbf{x}}) \rho^{*}\left(t, \overline{\mathbf{x}}^{\prime}\right) \frac{\left(\overline{\mathbf{x}} \times \overline{\mathbf{x}}^{\prime}\right)\left(\overline{\mathbf{x}} \times \overline{\mathbf{x}}^{\prime}\right)^{j}}{\left|\overline{\mathbf{x}}-\overline{\mathbf{x}}^{\prime}\right|^{3}} d \bar{x}^{\prime 3} d \bar{x}^{3}
\end{gathered}
$$

$\left(\overline{\mathbf{x}} \times \overline{\mathbf{x}}^{\prime}\right)^{i}=\varepsilon_{i j k} \bar{x}^{j} \bar{x}^{\prime k}$, and $\bar{V}$ is the support of the body in the $\mathrm{RS}^{+}$. When deriving (19)-(29) we supposed that (15) is valid in the RS. Let us note that $\bar{Q}^{k}$ (and, therefore, $\bar{S}^{i}$ ) is explicitly proportional to the matter velocity $\dot{\bar{x}}^{i}$ relative to the $\mathrm{RS}^{+}$. Moreover, $\bar{Q}^{k}$ and $\bar{S}^{i}$ have the same functional form as the original spin density $Q^{k}$ and the spin $S^{i}$ relating to the RS (see, (12)-(16)). Not pretending to a rigorous physical meaning, we can call $\bar{S}^{i}$ "post-Newtonian angular momentum of the body relative to the RS" ${ }^{+}$. On the other hand, the matrix $\bar{C}^{i j}$, being symmetric $\bar{C}^{i j}=\bar{C}^{j i}$ can be called "post-Newtonian tensor of inertia in the RS ${ }^{+}$". 


\section{Post-Newtonian Tisserand axes and principal axes of inertia}

It is to note that although all the quantities entering (19) contain explicit post-Newtonian terms, (19) itself looks formally analogous to the Newtonian rotational equations of motion relative to a rigidly rotating reference system. This allows us to introduce the post-Newtonian Tisserand axes and the principal axes of inertia in a formally Newtonian way as it was done, e.g., in Moritz, Mueller (1987). Thus we can define

- The post-Newtonian Tisserand reference system $\mathrm{RS}_{1}^{+}$by imposing the condition

$$
\bar{S}^{i}=0 \text {. }
$$

- The post-Newtonian reference system of principal axes of inertia $\mathrm{RS}_{2}^{+}$ by imposing the condition

$$
\bar{C}^{i j}(t)=\operatorname{diag}(\mathcal{A}(t), \mathcal{B}(t), \mathcal{C}(t)) .
$$

Both (29) and (30) should be considered as the definitions of the rotational matrix $P^{i j}(t)$ which relates the RS and the $\mathrm{RS}^{+}$.

In the $\mathrm{RS}_{1}^{+}$the rotational equations of motion (19) read

$$
\frac{d}{d t}\left(\bar{C}^{i j} \bar{\omega}^{j}\right)+\varepsilon_{i j k} \bar{C}^{k s} \bar{\omega}^{j} \bar{\omega}^{s}=P^{i j} F^{j}+\mathcal{O}\left(c^{-4}\right) .
$$

The condition (29) which fixes the $\mathrm{RS}_{1}^{+}$can be expressed through the coordinates of the RS as

$$
\begin{gathered}
\varepsilon_{i j k} \int_{V} x^{j} \mathcal{Q}^{k} d x^{3}=0 \\
\mathcal{Q}^{k}=\rho^{*} V^{k}+\frac{1}{c^{2}}\left(p^{k s} V^{s}-\frac{1}{2} G \rho^{*} \int_{V} \rho^{*}\left(t, \mathbf{x}^{\prime}\right) V^{\prime s} \frac{7 \delta^{k s}+n^{k} n^{s}}{\left|\mathbf{x}-\mathbf{x}^{\prime}\right|} d x^{\prime 3}\right), \\
V^{i}=\dot{x}^{i}-\varepsilon_{i j k} \omega^{j} x^{k}, \quad V^{\prime i}=\dot{x}^{\prime i}-\varepsilon_{i j k} \omega^{j} x^{\prime k}
\end{gathered}
$$

where

$$
\omega^{i}=P^{j i} \bar{\omega}^{j}=\frac{1}{2} \varepsilon_{i j k} P^{m j} \dot{P}^{m k}
$$

is the angular velocity of rotation of the $\mathrm{RS}^{+}$relative to the $\mathrm{RS}$ projected onto the axes of the RS. Then (32)-(34) can be written as a system of linear algebraic equations defining $\omega^{i}$ at each moment of time

$$
\begin{gathered}
S^{i}(t)-C^{i j}(t) \omega^{j}(t)=0 \\
C^{i j}=P^{a i} P^{b j} \bar{C}^{a b}=\int_{V} \rho^{*}\left(\delta^{i j} x^{s} x^{s}-x^{i} x^{j}\right) d x^{3}+\frac{1}{c^{2}} \varepsilon_{i a k} \varepsilon_{j b s} \int_{V} x^{a} x^{b} p^{k s} d x^{3} \\
-\frac{G}{2 c^{2}}\left(7 \delta^{i j} \alpha^{s s}-7 \alpha^{i j}+\beta^{i j}\right)
\end{gathered}
$$




$$
\begin{gathered}
\alpha^{i j}=P^{a i} P^{b j} \bar{\alpha}^{a b}=\int_{V} \int_{V} \rho^{*}(t, \mathbf{x}) \rho^{*}\left(t, \mathbf{x}^{\prime}\right) \frac{x^{i} x^{\prime j}}{\left|\mathbf{x}-\mathbf{x}^{\prime}\right|} d x^{\prime 3} d x^{3}, \\
\beta^{i j}=P^{a i} P^{b j} \bar{\beta}^{a b}=\int_{V} \int_{V} \rho^{*}(t, \mathbf{x}) \rho^{*}\left(t, \mathbf{x}^{\prime}\right) \frac{\left(\mathbf{x} \times \mathbf{x}^{\prime}\right)^{i}\left(\mathbf{x} \times \mathbf{x}^{\prime}\right)^{j}}{\left|\mathbf{x}-\mathbf{x}^{\prime}\right|^{3}} d x^{\prime 3} d x^{3},
\end{gathered}
$$

where $\left(\mathbf{x} \times \mathbf{x}^{\prime}\right)^{i}=\varepsilon_{i j k} x^{j} x^{\prime k}, S^{i}$ is defined by (12)-(16), and both $S^{i}$ and $C^{i j}=C^{j i}$ are obviously independent of $\omega^{i}$.

According to (36) the post-Newtonian spin $S^{i}=C^{i j} \omega^{j}$ and, therefore, it can be split into a product of the angular velocity $\omega^{i}$ and the tensor of inertia $C^{i j}$. Hence the rotational equations of motion (11) can be re-written as

$$
\frac{d}{d t}\left(C^{i j} \omega^{j}\right)=F^{i}
$$

$\omega^{i}$ being defined by (36).

It is interesting to note that the post-Newtonian tensor of inertia $C^{i j}$ defined by (37)-(39) formally coincides with the expression for the postNewtonian tensor of inertia derived in Soffel (1994) in the first postNewtonian approximation for an isolated, rigidly rotating body with the aid of the approach proposed by Thorne and Gürsel (1983). The approach used in Soffel (1994) accounts only for first-order terms in $\epsilon=\omega R / c$, where $R$ is typical linear size of the body (that is, the body is supposed to rotate slowly enough). On the contrary, we make use of the general post-Newtonian approximation scheme.

\section{The post-Newtonian torque and the BD moments}

In Voinov (1988), and Damour, Soffel and Xu (1993) it has been shown that the local reference system (GRS) is physically adequate for modelling rotational motion of a body being a member of an N-body system. From now on, we consider the "nonrotating" reference system $\left(t, x^{i}\right)$ used above to coincide with the GRS. In the latter of the two papers cited above it has been shown also that the post-Newtonian torque $F^{i}$ defined by (17) cannot be expressed through the Blanchet-Damour mass and spin moments of the body under consideration and that it is possible to define the postNewtonian spin as $\tilde{S}^{i}=S^{i}+c^{-2} S^{\prime i}$, where $S^{\prime i}$ is an additional term vanishing together with external potentials. Then the rotational equations of motion read

$$
\frac{d}{d t} \tilde{S}^{i}=\tilde{F}^{i}+\mathcal{O}\left(c^{-4}\right), \quad \tilde{F}^{i}=F^{i}+\frac{1}{c^{2}} \frac{d}{d t} S^{i},
$$

and $S^{i}$ can defined in such a way that

$$
\tilde{F}^{i}=\varepsilon_{i a b} \sum_{l=0}^{\infty} \frac{1}{l !}\left[M_{a L} G_{b L}+\frac{1}{c^{2}} \frac{l+1}{l+2} S_{a L} H_{b L}\right]+\mathcal{O}\left(c^{-4}\right),
$$


where $L=i_{1} i_{2} \ldots i_{l}$ is the multiindex, $M_{L}$ and $S_{L}$ are the BD mass and spin moments, and $G_{L}$ and $H_{L}$ are the electric- and magnetic type tidal moments of external gravitational field (see, Damour, Soffel, Xu (1993) for details).

Starting from (41) and applying the same arguments, which allowed us to derive (31) and (40) from (11), we can introduce another version of the post-Newtonian Tisserand reference system $\mathrm{RS}_{3}^{+}$. Making use of the $\mathrm{RS}_{3}^{+}$, the rotational equations of motion can be written as

$$
\begin{gathered}
\frac{d}{d t}\left(C^{i j} \tilde{\omega}^{j}\right)=\tilde{F}^{i}+\mathcal{O}\left(c^{-4}\right), \\
\frac{d}{d t}\left(\bar{C}^{i j} \overline{\tilde{\omega}}^{j}\right)+\varepsilon_{i j k} \bar{C}^{k s} \overline{\tilde{\omega}}^{j} \overline{\tilde{\omega}}^{s}=\tilde{P}^{i j} \tilde{F}^{j}+\mathcal{O}\left(c^{-4}\right) .
\end{gathered}
$$

where $\tilde{\omega}^{i}$ is defined by

$$
\tilde{S}^{i}(t)-C^{i j}(t) \tilde{\omega}^{j}(t)=0
$$

and the relations between angular velocities $\tilde{\omega}^{i}, \overline{\tilde{\omega}}^{i}$ and the corresponding orthogonal matrix $\tilde{P}^{i j}$ are the same as above (see, (20) and (35)).

The definition of angular velocity of a non-rigid body is not unique even in Newtonian physics (as we mentioned above one can introduce the Tisserand axes and the principal axes of inertia which give different definitions of angular velocity). In general relativity there is also another reason of the nonuniqueness: the post-Newtonian spin itself of a body being a member of an $\mathrm{N}$-body system is not unique due to contributions of the gravitational field binding the system (see, also Damour, Soffel, Xu (1993)). Considering this nonuniqueness of the angular velocity, the definitions (36) and (45) can be considered as conventions which make the laws of rotational motion as simple as possible.

\section{A note on astronomical applications}

We showed above that the post-Newtonian rotational equations of motion can be written in a formally Newtonian way. Therefore, many of the Newtonian results concerning the rotational motion of non-rigid bodies are also valid in the post-Newtonian approximation of general relativity. For example, if we suppose the body to be "dynamically rigid" in the $\mathrm{RS}^{+}$ $\bar{C}^{i j}=$ const then (31) or (44) are precisely equivalent to the Euler equations. The influence of general relativity results in the non-Newtonian expressions for the torque $\tilde{F}^{i}$ and for the tensor of inertia $C^{i j}$. As for the latter, one could try to derive a system of differential equations defining time-dependence of $C^{i j}$ and $\bar{C}^{i j}$ (related to the post-Newtonian hydrodynamical equations). However, since the internal structure of celestial bodies 
(including the Earth) is not known well enough to obtain the tensor of inertia with sufficient accuracy by evaluating the integrals (37)-(39) or the like, $C^{i j}$ is to be derived from various kinds of astronomical observations. Therefore, probably the most important difference between the post-Newtonian rotational equations of motion derived above and their Newtonian counterparts is the relativistic corrections to the torque. Eq. (31) can be used to derive relativistic effects in the forced polar motion. Eq. (43) can be used to investigate relativistic effects in the forced precession and nutation (see, Bizouard, et al. (1992)). More detailed evaluation of the astronomical consequences will be published elsewhere.

Acknowledgement. The author is indebted to Prof. M.Soffel and Dr. A.Voinov for insightful discussions.

\section{References}

Bizouard C., Schastok, J., Soffel M.H., Souchay J., (1992) Étude de la rotation de la Terre dans le cadre de la relativité général: premiere approche. In: Journées 1992, N. Capitaine (ed.), Observatoire de Paris, pp. 76-84

Brumberg, V.A. (1972) Relativistic Celestial Mechanics. Nauka, Moscow. (in Russian)

Brumberg, V.A., Kopejkin, S.M. (1989) Relativistic Theory of Celestial Reference Frames. In Reference Frames, edited by J. Kovalevsky, I.I. Mueller and B.Kolaczek. Kluwer Academic Publishers, pp. 115

Damour, T., Soffel, M., Xu, C. (1991) General Relativistic Celestial Mechanics I. Method and definition of reference systems Phys. Rev. D, Vol. no. 43, pp. 3273-3307

Damour, T., Soffel, M., Xu, C. (1992) General Relativistic Celestial Mechanics II. Translational Equations of Motion Phys. Rev. D, Vol. no. 45, pp. 1017-1044

Damour, T., Soffel, M., Xu, C. (1993) General Relativistic Celestial Mechanics III. Rotation Equations of Motion Phys. Rev. D, Vol. no. 47, pp. 3124-3137

Fock, V.A. (1959) Theory of space, time and gravitation. Pergamon, Oxford.

Klioner, S.A., Voinov, A.V. (1993) Relativistic Theory of Astronomical Reference Systems in Closed Form. Phys. Rev. D, Vol. no. 48, pp. 1451-1461

Kopejkin, S.M. (1988) Celestial Coordinate Reference Systems in Curved Space-Time. Celestial Mechanics, Vol. no. 44, pp. 87-115

Landau, L.D., Lifshitz, E.M. (1971) The Classical Theory of Fields. Pergamon Press, Oxford.

Moritz, H., Mueller, I.I. (1987) Earth Rotation: Theory and Observation Ungar, New York.

Soffel, M. (1994) The problem of rotational motion and rigid bodies in the post-Newtonian framework. unpublished notes

Synge, J.L. (1960) Relativity: the General Theory. North-Holland Publishing Company, Oxford.

Thorne, K.S., Gürsel, Y. (1983) The free precession of slowly rotating neutron stars: rigidbody motion in general relativity, Mon. Not. $R$ astr. Soc., Vol. no. 205, pp. 809-817

Voinov, A.V. (1988) Motion and rotation of celestial bodies in the post-Newtonian approximation, Celestial Mechanics, Vol. no. 41, pp. 293-307 